\title{
Vehicle Traffic Flow Detection System Based on Video Images under Haze Environment
}

\author{
Liu Jun-Fei ${ }^{1}$, Wu Jian-Zhen ${ }^{1}$, Li Hong-Qin ${ }^{1}$ \\ School of Electronic and Electrical Engineering, Shanghai University of Engineering Science, Shanghai ${ }^{1}$
}

\begin{abstract}
In order to study vehicle traffic flow under extreme weather conditions such as haze and so on, this paper mainly focus on the application of related video processing technology in vehicle detection, and studies the traffic flow detection method based on video images. Based on the Gaussian mixture model, background detection and video de-haze algorithm is used to model the background, video frame is extracted and HSV model is used to make the video image clearer and then traffic flow is detected. MATLAB simulation results show that the proposed flow detection system can produce high detection accuracy of the vehicle in the haze environment.
\end{abstract}

Keywords: Gauss model, HSV model, de-haze algorithm, vehicle detection and tracking.

\section{INTRODUCTION}

With the upgrading of China's economic base, the degree of industrialization gradually increased, air quality began to deteriorate, haze weather phenomenon increased, the adverse effects on modern intelligent traffic management is increasing. In order to solve the increasingly serious traffic problems under haze and other extreme weathers, research on vehicle traffic flow is significantly increase ${ }^{[1-2]}$. The system is a kind of intelligent transportation system which uses many kinds of high-tech, such as computer technology, database technology and image processing technology. The traffic flow parameters, such as vehicle flow rate, vehicle speed and vehicle type, are the basis of signal control, traffic simulation and decision making, and play an important role in the intelligent transportation system. Vehicle detection and tracking plays an important role in intelligent transportation system.

\section{Haze image Enhancement based on Haze Concentration Estimation}

In order to obtain a clear video image, we first need to defog the video image. Due to different haze concentration, treatment methods will be different, so we need to distinguish between haze concentrations. RGB image is transformed into the HSV model ${ }^{[3]}$, the hue, saturation and brightness values of the image are analyzed. Then the haze concentration is determined according to the $\mathrm{V}$ component distribution range threshold in the HSV model. If it is greater than the maximum threshold $[0,0,0.7529]$, the image is normal image without haze and can be directly outputted; if it is less than the maximum threshold, but greater than the minimum threshold $[0,0,0.4118]$, the image is a mist or thick haze image; if it is less than the minimum threshold $[0,0,0.4118]$, then the image is a thick haze or fog image.

RGB values are normalized to the $[0,1]$ interval, and the HSI algorithm is consistent with the same algorithm. The formula can be presented as follows:

$$
\begin{gathered}
V=\max \\
S=\frac{\max -\min }{\max } \\
H^{\prime}=\left\{\begin{array}{c}
\frac{G-B}{\max -\min } \quad \text { if } \max =R \\
\frac{B-R}{\max -\min }+2 \quad \text { if } \max =G \\
\frac{R-G}{\max -\min }+4 \quad \text { if } \max =B \\
H=H^{\prime} * 60^{\circ} \\
H=H^{\prime}+360^{\circ} \quad \text { if } H^{\prime}<0
\end{array}\right.
\end{gathered}
$$

Where

$\max =\max (R, G, B)$

$\min =\min (R, G, B)$

For the thick haze image, we can transform the RGB model of video image into the HSI model to improve the value of I component in HSI model, and convert the HSI model into RGB model. While for thick haze or fog image, the dark channel image should be preliminary enhanced followed by local enhanced correction. The dark channel prior ${ }^{[4]}$ suggests that there are at least some pixels in the local area of most outdoor fog-free images with a low intensity value for one color channel. By simultaneously minimizing the $R, G$ and $B$ three channels of each pixel block, the $J_{\text {dark }}$ can be obtained.

$$
J_{\text {dark }}(x)=\min _{x \in(r, g, b)}\left(\min _{y \in \Omega(x)}\left(J_{c}(y)\right)\right)
$$

Where $\mathrm{J}_{\text {dark }}$ is the dark color of image $\mathrm{J}$, whose intensity value is close to $0 ; J_{c}$ is one of $R, G$ and $B$ channels of 
image $\mathrm{J} ; \Omega_{\mathrm{x}}$ is a regional centered on $\mathrm{x}$, it is assumed that to be a background point, the point is set to 0 , thus the the image block has the same depth. In fogged images, the moving target and background images can be extracted. intensity of these dark primary colors becomes higher, providing a good way to enhance dark primary colors. The The mixed Gaussian model is based on the background processed image can then be re-verified to output enhanced representation of the statistical information of pixel video.

The related formulas of HSI model ${ }^{[5]}$ are as follows:

$$
\begin{gathered}
I=\frac{1}{2}(\max +\min ) \\
S=\left\{\begin{array}{l}
\frac{\max -\min }{\max +\min }, \quad 0<I<=\frac{1}{2} \\
\frac{\max -\min }{2-(\max +\min )} \quad, \quad I>\frac{1}{2}
\end{array}\right. \\
H^{\prime}=\left\{\begin{array}{l}
\frac{G-B}{\max -\min } \quad \text { if } \max =R \\
\frac{B-R}{\max -\min }+2 \quad \text { if } \max =G \\
\frac{R-G}{\max -\min }+4 \quad \text { if } \max =B \\
H=H^{\prime} * 60^{\circ} \\
H=360^{\circ} \quad \text { if } H^{\prime}<0
\end{array}\right.
\end{gathered}
$$
samples. It uses the statistical information such as the probability density of a large number of sample values over a long period of time to represent the background, and then uses the statistical difference to judge the target pixel, and can carry out complex dynamic background modeling. In the mixed Gaussian background model, the color information between pixels is considered to be uncorrelated, and the processing of each pixel is independent of each other. For each pixel in the video image, the change of its value in the sequence image can be regarded as a stochastic process of generating the pixel value, that is, the Gaussian distribution is used to describe the color rendering rule of each pixel. For a multimodal Gaussian distribution model, each pixel of an image is modeled by a superposition of multiple Gaussian distributions of different weights, each Gaussian distribution corresponding to a state that may produce the color of the pixel, the weight of each Gaussian distribution and the distribution parameters are updated over time. The differential effect is shown in Fig.1.

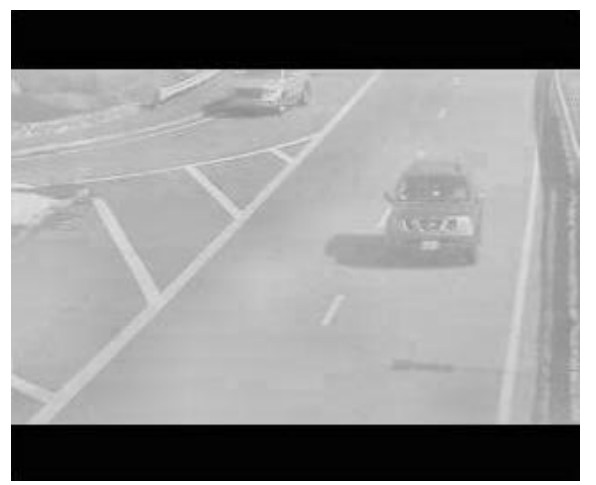

(a) Removal of the fog video frame

\section{Vehicle Traffic Flow System based on Video Processing Technology}

The enhanced video which has been processed by the haze concentration estimation is used to extract the image frame by frame and to perform the grayscale processing. If necessary, we can further do the gray-scale image de-noising process using smoothing and median filter. The purpose of the smoothing operation is to eliminate or minimize the noise and improve the quality of the image. The median filter is a sliding window with odd points in the neighborhood pixels sorted by gray-scale, the middle values will be used as the output pixels. The real-time and accuracy of the system lies in the tracking part. Select the center and color of the vehicle, these two features will be used as a track matching object, only when meet the conditions of these two features, it is considered to be the same car.

\subsection{Moving Vehicle Detection Technology}

The background subtraction method ${ }^{[6]}$ is used in this part. Background subtraction is also called background subtraction. The basic principle is to determine a background image as a reference image, and then subtract the current frame from the background frame to get the difference image, then use the threshold value $\mathrm{T}$ to determine the point in the image at current time which has a big difference from the gray value in the background model. If the difference is greater than $\mathrm{T}$, it is considered to be a target, and the point is set to 1 ; or it will be considered

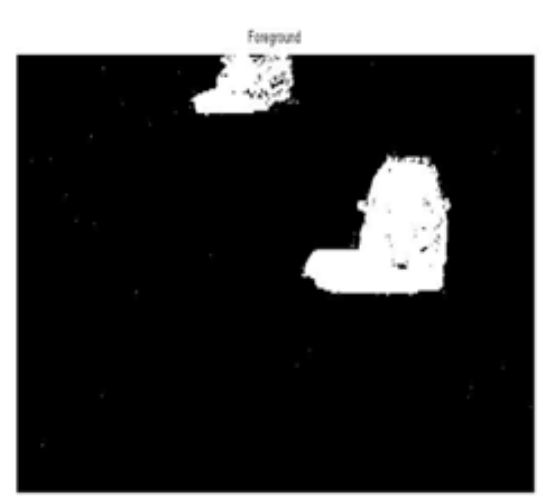

(b) Effect image of background difference Fig. 1 Moving Vehicle Detection

\subsection{Vehicle Tracking and Traffic Flow Counting}

Moving target tracking ${ }^{[7]}$ refers to the real-time tracking of the motion track of the monitored object through the static and dynamic characteristics in the video surveillance area, and establishes the correspondence relationship between 
the moving objects of the adjacent frame to obtain 1.3 Traffic Flow Counting

information such as the moving object position, movement After the moving object is extracted, for the real-time speed and movement trajectory.

traffic detection, the detection area is set on the image and the traffic flow is counted in the area instead of counting

On the basis of the video image processing and adjacent on the whole image. Common traffic flow counting frame processing, any moving object is tracked by the methods include virtual coil, and etc.

moving trajectory. In other words, establishing a corresponding match on target moving between adjacent The principle of the traffic flow counting using the frames, and uses it to determine whether the extracted detection line is as follows: first scan the pixels on the targets are the same. detection line in real-time, when continuous motion pixels (binary image in the white area) are discovered, it is In order to make the vehicle foreground has some considered that car is passing detection line at this time. characteristics, it is a common practice to cluster the pixels When the pixels on corresponding position turn into a belonging to the same vehicle region into blocks by using non-moving pixels (binary image in the black area), that the connectivity domain characteristic, and to express the means the vehicle has been through the detection line. characteristics of the vehicle by the rectangular blocks. To calculate the outer box of the rectangular block, we should find the outline of the foreground (target).

\section{TRAFFIC FLOW DETECTION SYSTEM DESIGN AND IMPLEMENTATION}

The basic idea of traffic flow detection system is to use the The proposed system is mainly aim to different haze background difference method to effectively detect the concentration environment, use the mobile vehicles as the movement of vehicles. Firstly, the Gaussian method is used detection target, install the camera on the top of the lane to to extract the background image. Secondly, the background obtain the necessary information related to the detection of frame is subtracted from each frame image to obtain the the important traffic real-time video, including video continuous motion frame of the foreground vehicle. The acquisition, haze mobile The target vehicle is detected in second part is to gray and binarize the video frame, and the video frame of the monitored object, and the haze, the then get the upper and lower boundary of the moving target haze concentration judgments, vehicles tracking and and the left and right boundary by computing the connected counting. The work flow of vehicle traffic flow detection domain of the binarization result, and then determine the system is shown in Fig.2:

tracking border to achieve the purpose of video tracking.

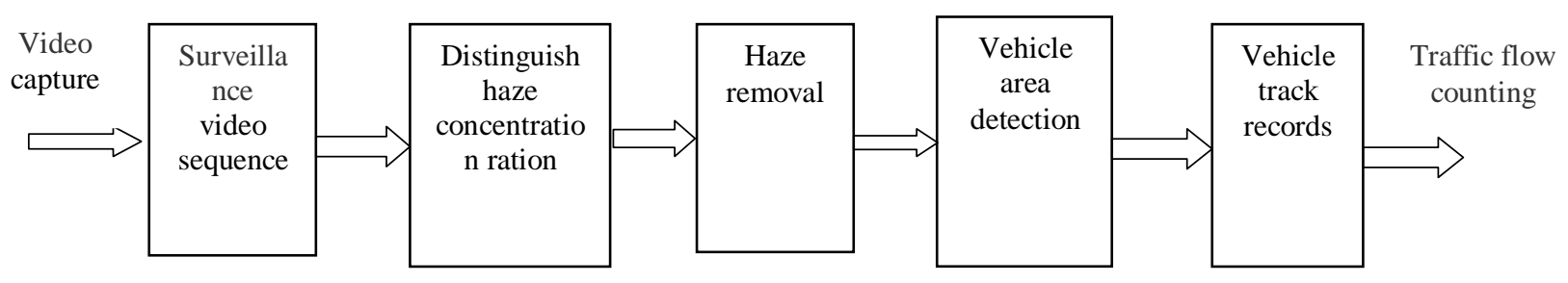

Fig. 2 Work flow of video vehicle detection system

The functions of each unit in Fig. 2 are as follows:

(1) Surveillance video sequence: video sequence captured by the camera is transferred to the computer to obtain video data, and save and waiting for processing;

(2) Distinguish haze concentration: the hue, saturation and brightness is obtained from the HSV model to distinguish haze concentration;

(3) Haze removal: video sequence is de-hazed and enhanced by the HSI algorithm and the dark color enhancement algorithm;

(4) Vehicle area detection: the upper and lower, left and right boundaries of the target area are determined by the marked connected region;
(5) Vehicle tracking records: trajectory of mobile vehicle in monitoring scene is under real-time tracking, and constantly moving vehicles is marked with a rectangular box.

\section{MATLAB SIMULATION RESULTS}

The flow chart of program design is shown in Fig.3. The example simulation results are shown in Fig.4 and Fig 5.

Once the rectangular frame is tracked on the road, the number will be displayed in the upper left corner of the display.

We use a video including 7 cars under thin and thick haze conditions and the simulation results show that the detected number of cars was 7 , so the proposed system can achieve the desired goal. 


\section{International Advanced Research Journal in Science, Engineering and Technology}

ISO 3297:2007 Certified

Vol. 3, Issue 10, October 2016

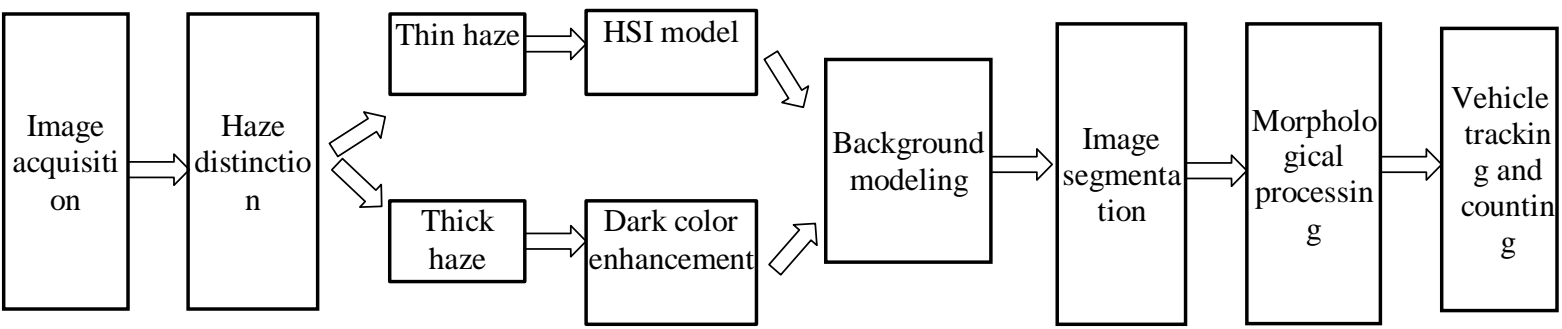

Fig. 3 Flow chart of program design

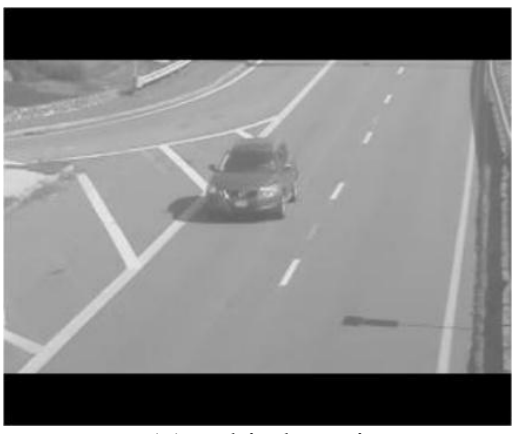

(a) thin haze image

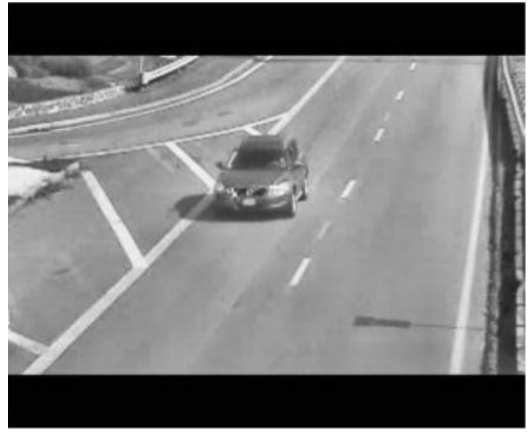

(b) haze removal

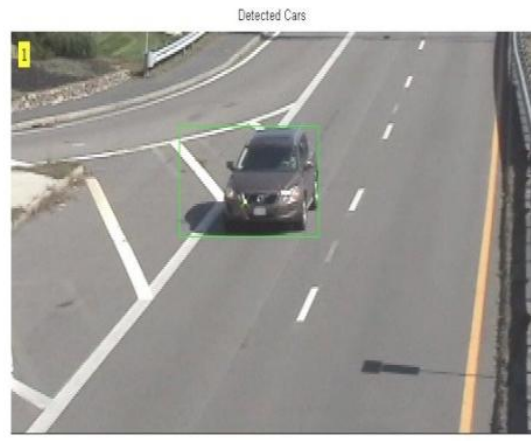

(c) vehicle tracking

Fig. 4 Example for vehicle flow detection based on thin haze video image

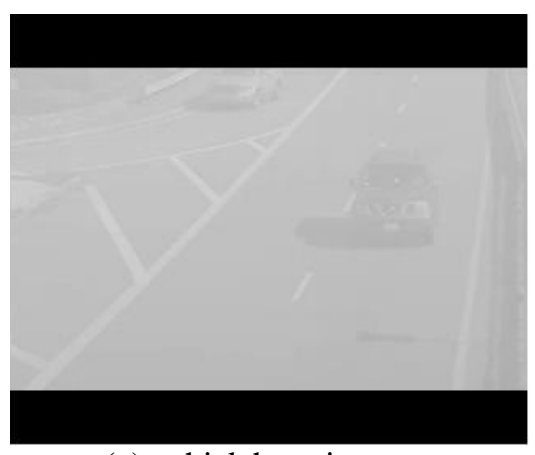

(a) thick haze image

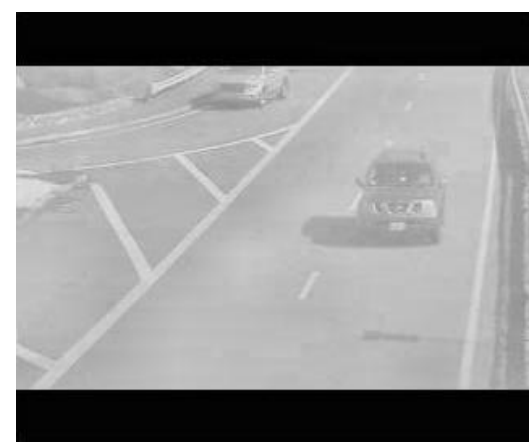

(b) haze removal

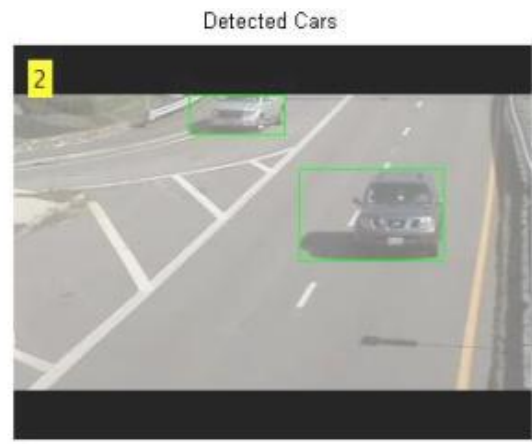

(c) Vehicle tracking

Fig. 5 Example for vehicle flow detection based on thick haze video image

\section{CONCLUSIONS}

In this paper, we proposed a traffic flow detection system based on video images under different haze concentration. The system uses hybrid Gaussian model, HSV model combined with video de-haze algorithm to clarify the video image so as to establish background model and vehicle tracking and counting. The results of simulations show that the proposed system can detect moving vehicles under different haze concentration with high accuracy.

\section{REFERENCES}

[1] Yen-Lin Chen, et al. A Vision-Based Driver Nighttime Assistance and Surveillance System Based on Intelligent Image Sensing Techniques and a Heterogamous Dual-Core Embedded System Architecture. Sensors, vol.12, No.3, pp.2373-99, 2012.

[2] Peng Y, Zhang Y, Hong Y. Day and night vehicle detection and counting in complex environment. International Conference of Image and Vision Computing, pp.453-458, 2013.
[3] Zhou Ming, Li Suzhen, Huo family. Moving target detection and tracking based on HSV model. Command Control \& Simulation, Vol.32, No.2, pp. 93-96, 2010.

[4] Jiang Jianguo, HouTianfeng, Qi Meibin. Improved image based on dark channel prior to fog algorithm. Journal of Circuits and Systems, Vol.16, No.2, pp.7-12, 2011.

[5] Wang Jianxin, Wang Zhiwei, Zhang, Zhang Jing, Li Juan. Single image defogging algorithm based on HSI color space. Computer Application, Vol.34, No.19, pp.2990-2995, 2014.

[6] Wang Guoqiang, Gaiqilin. Video target detection algorithm based on the method of background differences, Journal of Heilongjiang Hydraulic Engineering College, No.4, pp.64-68, 2014

[7] Ma xiubo. Research on vehicle detection and tracking technology based on video. Nankai University, 2013. 